\title{
Contribution of Microclimate towards Yield Attributing Factors and Yield of Summer Baby Corn (Zea mays L.) under Different Irrigations and Mulches
}

\author{
Trisha Manna ${ }^{1}$, G. Saha ${ }^{1}$, A. Saha ${ }^{2}$, D. Dutta ${ }^{2}$ and M.K. Nanda ${ }^{1}$ \\ ${ }^{1}$ Department of Agricultural Meteorology and Physics, B.C.K.V, Nadia, West Bengal-741252 \\ ${ }^{2}$ Department of Agronomy, B.C.K.V, Nadia, West Bengal-741252, India \\ *Corresponding author
}

\section{A B S T R A C T}

Keywords

Baby corn,

Absorbed PAR

(APAR), Light use

efficiency (LUE),

Irrigation, Mulch

Article Info

Accepted:

10 June 2018

Available Online:

10 July 2018
Field experiment was conducted at instructional farm, Jaguli, Bidhan Chandra Krishi Viswavidyalaya, Mohanpur, Nadia, West Bengal, during pre-kharif seasons of 2016 and 2017 to study crop microclimatic influence towards different yield attributing factors and yield of summer baby corn (Zea mays L.) var. G5414 $\mathrm{F}_{1}$-hybrid under irrigation levels like IW: CPE ratio at 1.0, 0.8 and 0.6 in main plot and mulches like no- mulch (control), polythene mulch, paddy straw mulch and jute mulch in sub plot under split-plot design with 3 replications. The results indicated that absorbed PAR $\left(\mu \mathrm{mol} \mathrm{m} \mathrm{sec}^{-1}\right)$ was higher for IW: CPE 1.0 and with paddy straw mulch. Light use efficiency $\left(\mathrm{g} / \mu \mathrm{mol} \mathrm{m} \mathrm{mec}^{-1}\right)$ showed highest values for IW: CPE 0.8 with polythene mulch. Yield attributes like cob length and weight with and without husk were found maximum with IW: CPE 1.0 and polythene mulch both the years. Highest baby corn yield $(1795 \mathrm{~kg} / \mathrm{ha})$ was achieved in combination of IW: CPE 1.0 and polythene mulch treatment. The APAR and LUE were measured at certain intervals for both the years and found to be positively correlated with different yield forming factors and yield in most of the times.

\section{Introduction}

Maize (Zea mays L.) is one of the highest yielding grain crops in the world following rice and wheat, but recently it has been cultivated as vegetable crop such as baby corn and time to time it finds a significant place as human food, animal feed, fodder etc. (Sahoo and Mahapatra, 2007). The productivity of summer corn is quite higher due to better water management that will help for achieving maximum yield per unit water application with minimal unavoidable losses of water because total number of irrigation applied is not so important than timely and adequate application of water. Reduction of evapotranspiration from soil using different mulches offers possibility of increasing the use efficiency of water by the crop. In comparison with conventional tillage and mulching in maize, mulching has the more moisture retention capability (Jones et al., 1969). Both irrigation and mulching modifies the crop microclimate that will effect in 
various physical, chemical and physiological changes in crop plant, affecting crop growth, productivity and yield. With this background, our present study concentrated on microclimatic effect towards better yield contributing factors, yield attributes and yield of summer baby corn.

\section{Materials and Methods}

Site description, crop management and treatment details

Two years field experiment was undertaken in the pre-kharif seasons of 2016 and 2017 at Instructional Farm, Jaguli, Bidhan Chandra Krishi Viswavidyalaya, Mohanpur, Nadia, W.B. (Latitude: $22^{\circ} 56^{\prime} \mathrm{N}$, Longitude: $88^{\circ} 32^{\prime}$ E and Altitude: $9.75 \mathrm{~m}$ above mean sea level) with three levels of irrigations at IW: CPE ratios of $1.0\left(\mathrm{I}_{1}\right), 0.8\left(\mathrm{I}_{2}\right), 0.6\left(\mathrm{I}_{3}\right)$ treated as main plot and four levels of mulching: control or no mulch $\left(\mathrm{M}_{0}\right)$, polythene mulch,30 $\mu\left(\mathrm{M}_{1}\right)$, paddy straw mulch@5t ha ${ }^{-1}\left(\mathrm{M}_{2}\right)$ and jute mulch@500 gsm $\left(\mathrm{M}_{3}\right)$ as sub plot factors with three replications in split-plot design. Seeds @ $20 \mathrm{~kg} \mathrm{ha}^{-1}$ are sown in lines with spacing of 40 $\mathrm{cm} \times 20 \mathrm{~cm}$ in raised beds of $60 \mathrm{~cm}$ width on $19^{\text {th }}$ of February both the years and maintained irrigation depth was $5 \mathrm{~cm}$. First common irrigation was applied at the date of sowing and subsequent irrigations were applied as per individual treatments. 10 tonnes of $\mathrm{FYM}+$ 120:60:40 N: $\mathrm{P}_{2} \mathrm{O}_{5}: \mathrm{K}_{2} \mathrm{O} \mathrm{kg} / \mathrm{ha}$ applied as basal to all plots.

\section{Observations of microclimatic parameters}

Daily evaporation was measured from USWB open class A pan evaporimeter situated at Principle Agrometeorological Observatory, Jaguli, Mohanpur and accumulated to determine the next date of irrigation (climatological irrigation scheduling). Periodical observations of different components of photosynthetically active radiation (PAR) started from 20 DAS at 11:30 am to $12: 30 \mathrm{pm}$ with line quantum sensor (Model: LI -191R with LI-250A light meter). Total incoming and canopy reflected PAR was measured above the canopy; transmitted and soil reflected PAR was measured at 6" above soil surface.

Absorbed PAR was worked out by the following equation -

$A P A R\left(\mu \mathrm{mol} \mathrm{m}^{-2} \mathrm{~s}^{-1}\right)=\left(I P A R+R P A R_{s}\right)-\left(T P A R+R P A R_{c}\right)$

Where,

$\mathrm{R}=$ Intercepted PAR above the canopy, TPAR = Transmitted PAR,

$\mathrm{RPAR}_{\mathrm{c}}=$ Canopy reflected PAR, $\mathrm{RPAR}_{\mathrm{s}}=$ Soil reflected PAR

Light use efficiency (LUE) can be estimated using the following formula-

$L U E\left(g / \mu \mathrm{mol} \mathrm{m}^{-2} \mathrm{~s}^{-1}\right)=\frac{\text { Amountofdry matter produced }\left(\mathrm{g} / \mathrm{m}^{2}\right)}{\text { Amount of cumulative PAR absorbed }\left(\mu \operatorname{molm} \mathrm{m}^{-2}{ }^{-1}\right)}$

\section{Observations of biometric parameters}

A periodical observation of total biomass of baby corn was done at 20,30, 40, 50 and 60 DAS. The crop was harvested by periodical plucking of green cobs starting from 50 DAS with frequency 3. After harvesting of fresh cobs, husked and dehusked cob length was measured by centimetre scale or ruler and weight was taken in electrical balance. After 3 plucking corn yield was estimated in $\mathrm{kg} \mathrm{ha}^{-1}$.

\section{Statistical analysis}

The data on different aspects of baby corn were subjected to statistical analysis by using the technique of analysis of variance (ANOVA) as suggested by Gomez and Gomez (1984).The significance of differences for treatments was tested by " $F$ " test at $5 \%$ level. The critical differences were calculated when differences among the treatments were found significant by " $F$ " test. 


\section{Results and Discussion}

Effect of irrigation and mulching on total accumulated biomass $\left(\mathrm{g}\right.$ plant $\left.^{-1}\right)$ of baby corn

Figure 1 and 2 shows the effect of irrigation and mulching on total accumulated biomass ( $\mathrm{g}$ plant $^{-1}$ ) of baby corn (pooled data). Accumulated biomass was maximum in IW: CPE $1.0\left(\mathrm{I}_{1}\right)$ at 60 DAS followed by IW: CPE $0.8\left(\mathrm{I}_{2}\right)$ and IW: CPE $0.6\left(\mathrm{I}_{3}\right)$. Biomass accumulation rate was slower from 20 to 30 DAS and the difference due to different treatments was very close to each other. Narang et al., 1989 also reported higher dry matter plant ${ }^{-1}$ with lower CPE value due to less water stress, more availability of nutrients and good vigour of plants. For mulching polythene mulch $\left(\mathrm{M}_{1}\right)$ at 60 DAS produced maximum biomass whereas there was not much variation in biomass production in paddy straw mulch $\left(\mathrm{M}_{2}\right)$ and no mulch $\left(\mathrm{M}_{0}\right)$ treatments. It is clear from the diagram that from 20 to 40 DAS there was not much variation in biomass accumulation in no mulch $\left(\mathrm{M}_{0}\right)$, paddy straw mulch $\left(\mathrm{M}_{2}\right)$ and jute mulch $\left(\mathrm{M}_{3}\right)$. Total accumulated biomass in root, shoot, and leaves was highly influenced by application of mulch due to moisture storage in soil for long duration after irrigation and proper nutrient utilization (Boonpradeep and Chatasiri, 1999).

Absorbed PAR ( $\mu$ mol m $\left.{ }^{-2} \sec ^{-1}\right)$ affected by irrigation and mulching of baby corn

Periodical data of absorbed photosynthetically active radiation during 2016 and 17 as influenced by the treatments are presented in Table 1. Maximum APAR of $1170.21 \mu \mathrm{mol}$ $\mathrm{m}^{-2} \mathrm{sec}^{-1}$ was observed at 50 DAS (pooled) for IW: CPE $1.0\left(\mathrm{I}_{1}\right)$ and minimum of 156.79 $\mu \mathrm{mol} \mathrm{m} \mathrm{sec}^{-1}$ was recorded in IW: CPE 0.8 $\left(\mathrm{I}_{2}\right)$. Adequate supply of moisture under $\mathrm{I}_{1}$ increased LAI, plant growth; thereby increased APAR. Water deficit may reduce the interception of solar radiation by maize crops due to the rolling up of leaves (Müller and Bergamaschi, 2005). Maximum APAR was observed for paddy straw mulch $\left(\mathrm{M}_{2}\right)$ amounting $1107.79 \mu \mathrm{mol} \mathrm{m} \mathrm{sec}^{-1}$ which was found statistically at par with control plots $\left(\mathrm{M}_{0}\right)$ at 50 DAS. The minimum APAR of $172.96 \mu \mathrm{mol} \mathrm{m} \mathrm{m}^{-2} \mathrm{sec}^{-1}$ was recorded in polythene mulch $\left(\mathrm{M}_{1}\right)$ at $20 \mathrm{DAS}$. Mulch, by reducing the soil water loss by evaporation, thus leaving more water available in the soil for transpiration (Moitra et al., 1996) for which leaf stomata remain opened and radiation absorption is better.

Effect of irrigation and mulching on light use efficiency $\left(\mathrm{g} / \mu \mathrm{mol} \mathrm{m} \mathrm{mec}^{-1}\right)$ of baby corn

Light use efficiency (LUE) establishes a good relationship with total accumulated biomass and APAR. The data regarding LUE at particular intervals in 2016 and 17 is presented in Table 2. The results revealed that IW: CPE $0.8\left(\mathrm{I}_{2}\right)$ at 60 DAS recorded maximum LUE $\left(0.162 \mathrm{~g} / \mu \mathrm{mol} \mathrm{m} \mathrm{sec}^{-1}\right.$, pooled $)$ and minimum LUE $\left(0.024 \mathrm{~g} / \mu \mathrm{mol} \mathrm{m} \mathrm{sec}^{-1}\right)$ was recorded at 30 DAS by IW: CPE $0.6\left(\mathrm{I}_{3}\right)$.

Irrigation recorded significant variation of LUE except at 30 DAS, 2016. LUE increased in deficit irrigation due to modification of PAR. Similar results were focused by Han et al., 2008 and $\mathrm{Li}$ et al., 2008a. Polythene mulch $\left(\mathrm{M}_{1}\right)$ during 60 DAS recorded highest LUE $\left(0.160 \mathrm{~g} / \mu \mathrm{mol} \mathrm{m} \mathrm{m}^{-2} \mathrm{sec}^{-1}\right)$ and lowest value $\left(0.026 \mathrm{~g} / \mu \mathrm{mol} \mathrm{m} \mathrm{mec}^{-1}\right)$ recorded with jute mulch $\left(\mathrm{M}_{3}\right)$ at 30 DAS (pooled) which was statistically at par with paddy straw mulch $\left(\mathrm{M}_{2}\right)$ at the same time. Except 40 DAS in 2016, effect of mulching on LUE was statistically significant. Interaction effect $(\mathrm{I} \times \mathrm{M})$ was also significant in all cases except at 40 DAS in 2016. 
Table.1 Absorbed PAR ${ }^{\mathrm{nol} \mathrm{m}} \mathrm{m}^{-2} \mathrm{~s}^{-1}$ ) influenced by irrigation and mulching in baby corn

\begin{tabular}{|c|c|c|c|c|c|c|c|c|c|c|c|c|c|c|c|c|}
\hline \multirow{2}{*}{\multicolumn{2}{|c|}{ Treatments }} & \multicolumn{2}{|c|}{20 DAS } & \multirow[b]{2}{*}{ Pooled } & \multicolumn{2}{|c|}{30 DAS } & \multirow[b]{2}{*}{ Pooled } & \multicolumn{2}{|c|}{40 DAS } & \multirow[b]{2}{*}{ Pooled } & \multicolumn{2}{|c|}{50 DAS } & \multirow[b]{2}{*}{ Pooled } & \multicolumn{2}{|c|}{60 DAS } & \multirow[b]{2}{*}{ Pooled } \\
\hline & & 2016 & 2017 & & 2016 & 2017 & & 2016 & 2017 & & 2016 & 2017 & & 2016 & 2017 & \\
\hline \multicolumn{2}{|c|}{$I_{1}(I W: C P E=1.0)$} & 276.63 & 277.28 & 276.96 & 307.02 & 290.52 & 298.77 & 677.25 & 959.73 & 818.49 & 1166.44 & 1173.97 & 1170.21 & 1116.69 & 1099.13 & 1107.91 \\
\hline \multicolumn{2}{|c|}{$I_{2}(I W: C P E=0.8)$} & 155.79 & 157.79 & 156.79 & 376.01 & 303.58 & 339.79 & 564.35 & 588.45 & 576.40 & 1077.31 & 1086.70 & 1082.01 & 592.90 & 640.65 & 616.78 \\
\hline \multicolumn{2}{|c|}{$I_{3}(I W: C P E=0.6)$} & 186.12 & 189.97 & 188.05 & 486.53 & 513.51 & 500.02 & 778.27 & 997.75 & 888.01 & 919.43 & 915.46 & 917.44 & 667.68 & 682.95 & 675.32 \\
\hline \multicolumn{2}{|c|}{$\operatorname{SEm}( \pm)$} & 0.02 & 1.1952 & 0.61 & 31.48 & 10.781 & 21.13 & 102.81 & 6.0544 & 54.43 & 15.08 & 16.33 & 15.70 & 0.23 & 3.86 & 2.04 \\
\hline \multicolumn{2}{|c|}{ CD (at 5\%) } & 0.07 & 4.6921 & 2.38 & 123.58 & 42.325 & 82.95 & NS & 23.769 & 23.77 & 59.19 & 64.11 & 61.65 & 0.90 & 15.14 & 8.02 \\
\hline \multicolumn{2}{|c|}{$\mathbf{M}_{0}($ Control $)$} & 204.98 & 205.87 & 205.42 & 331.28 & 263.21 & 297.24 & 823.83 & 1089.3 & 956.58 & 1083.29 & 1109.38 & 1096.34 & 817.11 & 881.02 & 849.06 \\
\hline \multicolumn{2}{|c|}{$\mathbf{M}_{1}($ Polythene mulch) } & 172.16 & 173.75 & 172.96 & 296.28 & 297.21 & 296.75 & 608.19 & 890.61 & 749.40 & 975.94 & 948.94 & 962.44 & 747.45 & 778.56 & 763.00 \\
\hline \multicolumn{2}{|c|}{$\mathrm{M}_{2}$ (Paddy straw mulch) } & 227.10 & 232.63 & 229.86 & 393.84 & 445.24 & 419.54 & 647.18 & 800.92 & 724.05 & 1098.10 & 1117.48 & 1107.79 & 690.62 & 690.32 & 690.47 \\
\hline \multicolumn{2}{|c|}{$M_{3}($ Jute mulch $)$} & 220.48 & 221.14 & 220.81 & 538.01 & 471.14 & 504.58 & 613.96 & 613.71 & 613.84 & 1060.25 & 1059.03 & 1059.64 & 914.52 & 880.41 & 897.46 \\
\hline \multicolumn{2}{|r|}{$\operatorname{SEm}( \pm)$} & 0.34 & 1.4992 & 0.92 & 47.66 & 13.795 & 30.73 & 105.43 & 6.1139 & 55.77 & 19.64 & 15.95 & 17.79 & 0.20 & 2.74 & 1.47 \\
\hline \multicolumn{2}{|c|}{ CD (at 5\%) } & 1.01 & 4.4545 & 2.73 & 141.60 & 40.99 & 91.30 & NS & 18.166 & 18.17 & 58.35 & 47.39 & 52.87 & 0.60 & 8.15 & 4.37 \\
\hline \multirow[t]{2}{*}{$\mathbf{I} \times \mathbf{M}$} & $\operatorname{SEm}( \pm)$ & 0.59 & 2.5967 & 1.59 & 82.55 & 23.894 & 53.22 & 182.61 & 10.59 & 96.60 & 34.01 & 27.62 & 30.82 & 0.35 & 4.75 & 2.55 \\
\hline & CD (at 5\%) & 1.75 & 7.7153 & 4.73 & NS & 70.996 & 71.00 & NS & 31.464 & 31.46 & 101.07 & 82.08 & 91.57 & 1.04 & 14.11 & 7.57 \\
\hline
\end{tabular}


Table.2 LUE ${ }^{\prime} \mu \mathrm{mol} \mathrm{m}^{-2} \mathrm{~s}^{-1}$ ) of baby corn as affected by irrigation and mulching

\begin{tabular}{|c|c|c|c|c|c|c|c|c|c|c|c|c|c|c|c|c|}
\hline \multirow{2}{*}{\multicolumn{2}{|c|}{ Treatments }} & \multicolumn{2}{|c|}{20 DAS } & \multirow[b]{2}{*}{ Pooled } & \multicolumn{2}{|c|}{30 DAS } & \multirow[b]{2}{*}{ Pooled } & \multicolumn{2}{|c|}{40 DAS } & \multirow[b]{2}{*}{ Pooled } & \multicolumn{2}{|c|}{50 DAS } & \multirow[b]{2}{*}{ Pooled } & \multicolumn{2}{|c|}{60 DAS } & \multirow[b]{2}{*}{ Pooled } \\
\hline & & 2016 & 2017 & & 2016 & 2017 & & 2016 & 2017 & & 2016 & 2017 & & 2016 & 2017 & \\
\hline \multicolumn{2}{|c|}{$I_{1}(I W: C P E=1.0)$} & 0.034 & 0.035 & 0.035 & 0.044 & 0.060 & 0.052 & 0.092 & 0.046 & 0.069 & 0.038 & 0.061 & 0.050 & 0.076 & 0.116 & 0.096 \\
\hline \multicolumn{2}{|r|}{$I_{2}(I W: C P E=0.8)$} & 0.043 & 0.047 & 0.045 & 0.033 & 0.061 & 0.047 & 0.059 & 0.060 & 0.060 & 0.041 & 0.050 & 0.045 & 0.147 & 0.178 & 0.162 \\
\hline \multicolumn{2}{|r|}{$\mathrm{I}_{3}(\mathrm{IW}: \mathrm{CPE}=0.6)$} & 0.033 & 0.037 & 0.035 & 0.022 & 0.026 & 0.024 & 0.036 & 0.027 & 0.032 & 0.042 & 0.052 & 0.047 & 0.124 & 0.140 & 0.132 \\
\hline \multicolumn{2}{|r|}{$\operatorname{SEm}( \pm)$} & 0.001 & 0.001 & 0.001 & 0.005 & 0.003 & 0.004 & 0.007 & 0.000 & 0.003 & 0.001 & 0.002 & 0.001 & 0.000 & 0.001 & 0.000 \\
\hline \multicolumn{2}{|r|}{ CD (at 5\%) } & 0.003 & 0.002 & 0.003 & NS & 0.013 & 0.013 & 0.026 & 0.002 & 0.014 & 0.002 & 0.007 & 0.005 & 0.001 & 0.003 & 0.002 \\
\hline \multicolumn{2}{|r|}{$\mathbf{M}_{0}$ (Control) } & 0.033 & 0.033 & 0.033 & 0.032 & 0.068 & 0.050 & 0.039 & 0.025 & 0.032 & 0.033 & 0.042 & 0.038 & 0.101 & 0.115 & 0.108 \\
\hline \multicolumn{2}{|c|}{$\mathbf{M}_{1}($ Polythene mulch) } & 0.054 & 0.058 & 0.056 & 0.051 & 0.061 & 0.056 & 0.079 & 0.047 & 0.063 & 0.049 & 0.071 & 0.060 & 0.141 & 0.178 & 0.160 \\
\hline \multicolumn{2}{|c|}{$\mathbf{M}_{2}$ (Paddy straw mulch) } & 0.028 & 0.031 & 0.030 & 0.027 & 0.036 & 0.031 & 0.048 & 0.044 & 0.046 & 0.037 & 0.049 & 0.043 & 0.118 & 0.155 & 0.137 \\
\hline \multicolumn{2}{|r|}{$M_{3}$ (Jute mulch) } & 0.032 & 0.037 & 0.034 & 0.021 & 0.031 & 0.026 & 0.084 & 0.061 & 0.072 & 0.042 & 0.055 & 0.049 & 0.104 & 0.130 & 0.117 \\
\hline \multicolumn{2}{|r|}{$\operatorname{SEm}( \pm)$} & 0.000 & 0.001 & 0.001 & 0.005 & 0.004 & 0.004 & 0.017 & 0.000 & 0.008 & 0.001 & 0.002 & 0.002 & 0.000 & 0.001 & 0.001 \\
\hline \multicolumn{2}{|r|}{ CD (at 5\%) } & 0.001 & 0.003 & 0.002 & 0.014 & 0.011 & 0.012 & NS & 0.001 & 0.001 & 0.003 & 0.007 & 0.005 & 0.001 & 0.003 & 0.002 \\
\hline \multirow[t]{2}{*}{$\mathbf{I} \times \mathbf{M}$} & $\operatorname{SEm}( \pm)$ & 0.001 & 0.002 & 0.001 & 0.008 & 0.006 & 0.007 & 0.029 & 0.0005 & 0.015 & 0.002 & 0.004 & 0.003 & 0.0005 & 0.002 & 0.001 \\
\hline & CD (at 5\%) & 0.002 & 0.005 & 0.003 & 0.024 & 0.019 & 0.021 & NS & 0.001 & 0.001 & 0.004 & 0.012 & 0.008 & 0.001 & 0.005 & 0.003 \\
\hline
\end{tabular}


Table.3 Yield attributing factors of baby corn affected by irrigation and mulching

\begin{tabular}{|c|c|c|c|c|c|c|c|c|c|c|c|c|c|}
\hline \multirow{2}{*}{\multicolumn{2}{|c|}{ Treatments }} & \multicolumn{3}{|c|}{$\begin{array}{l}\text { Cob length }(\mathrm{cm}) \\
\text { (Husked) }\end{array}$} & \multicolumn{3}{|c|}{$\begin{array}{c}\text { Cob weight (g) } \\
\text { (Husked) }\end{array}$} & \multicolumn{3}{|c|}{$\begin{array}{l}\text { Corn length(cm) } \\
\text { (Dehusked) }\end{array}$} & \multicolumn{3}{|c|}{$\begin{array}{l}\text { Corn weight (g) } \\
\text { (Dehusked) }\end{array}$} \\
\hline & & 2016 & 2017 & Pooled & 2016 & 2017 & Pooled & 2016 & 2017 & Pooled & 2016 & 2017 & Pooled \\
\hline & $(I W: C P E=1.0)$ & 19.28 & 18.88 & 19.08 & 46.89 & 41.77 & 44.33 & 9.40 & 9.05 & 9.22 & 11.18 & 10.31 & 10.75 \\
\hline & $(\mathrm{IW}: \mathrm{CPE}=0.8)$ & 19.23 & 17.03 & 18.13 & 43.89 & 34.36 & 39.13 & 8.90 & 8.38 & 8.64 & 8.79 & 8.76 & 8.78 \\
\hline & $(I W: C P E=0.6)$ & 18.64 & 15.82 & 17.23 & 38.51 & 28.93 & 33.72 & 8.14 & 7.75 & 7.95 & 6.01 & 6.97 & 6.49 \\
\hline & $\operatorname{SEm}( \pm)$ & 0.08 & 0.16 & 0.12 & 0.18 & 0.24 & 0.21 & 0.16 & 0.10 & 0.13 & 0.29 & 0.07 & 0.18 \\
\hline & CD (at 5\%) & 0.31 & 0.63 & 0.47 & 0.71 & 0.96 & 0.83 & 0.61 & 0.39 & 0.50 & 1.13 & 0.27 & 0.70 \\
\hline & $\mathbf{M}_{0}$ (Control) & 15.92 & 13.79 & 14.86 & 38.31 & 30.68 & 34.49 & 7.20 & 7.09 & 7.15 & 6.50 & 7.43 & 6.97 \\
\hline $\mathbf{M}_{1}$ & Polythene mulch) & 21.87 & 18.69 & 20.28 & 48.00 & 39.60 & 43.80 & 8.99 & 10.08 & 9.54 & 10.21 & 10.55 & 10.38 \\
\hline $\mathbf{M}_{2}(\mathbf{I}$ & addy straw mulch) & 19.45 & 18.21 & 18.83 & 41.36 & 33.43 & 37.40 & 10.04 & 8.76 & 9.40 & 8.88 & 7.96 & 8.42 \\
\hline & $I_{3}$ (Jute mulch) & 18.97 & 18.27 & 18.62 & 44.71 & 36.37 & 40.54 & 9.03 & 7.63 & 8.33 & 9.05 & 8.78 & 8.92 \\
\hline & $\operatorname{SEm}( \pm)$ & 0.22 & 0.16 & 0.19 & 0.61 & 0.43 & 0.52 & 0.28 & 0.14 & 0.21 & 0.33 & 0.12 & 0.22 \\
\hline & CD (at 5\%) & 0.66 & 0.48 & 0.57 & 1.81 & 1.29 & 1.55 & 0.83 & 0.42 & 0.62 & 0.97 & 0.35 & 0.66 \\
\hline \multirow[t]{2}{*}{$\mathbf{I} \times \mathbf{M}$} & $\operatorname{SEm}( \pm)$ & 0.39 & 0.28 & 0.33 & 1.06 & 0.75 & 0.90 & 0.48 & 0.24 & 0.36 & 0.56 & 0.20 & 0.38 \\
\hline & CD (at 5\%) & 1.15 & 0.84 & 0.99 & 3.14 & 2.23 & 2.68 & 1.43 & 0.73 & 1.08 & 1.68 & 0.60 & 1.14 \\
\hline
\end{tabular}


Table.4 Baby corn yield $\left(\mathrm{kg} \mathrm{ha}^{-1}\right)$ of baby corn influenced by irrigation and mulching

\begin{tabular}{|c|c|c|c|c|}
\hline \multicolumn{2}{|c|}{ Treatments } & 2016 & \multirow{2}{*}{$\begin{array}{c}2017 \\
1502\end{array}$} & \multirow{2}{*}{$\begin{array}{c}\text { Pooled } \\
1505.61\end{array}$} \\
\hline & {$[\mathrm{W}: \mathrm{CPE}=1.0)$} & 1507 & & \\
\hline & $\mathrm{W}: \mathrm{CPE}=0.8)$ & 1454 & 1457 & 1455.88 \\
\hline & {$[\mathrm{W}: \mathrm{CPE}=0.6)$} & 1309 & 1315 & 1311.96 \\
\hline & $\operatorname{SEm}( \pm)$ & 21.69 & 18.47 & 20.08 \\
\hline & D (at 5\%) & 85.14 & 72.49 & 78.82 \\
\hline & $I_{0}($ Control $)$ & 1064 & 1079 & 1071.46 \\
\hline $\mathbf{M}_{1}$ & lythene mulch) & 1733 & 1728 & 1730.16 \\
\hline $\mathbf{M}_{2}(\mathbf{I}$ & ldy straw mulch) & 1347 & 1343 & 1345.32 \\
\hline & (Jute mulch) & 1550 & 1550 & 1549.67 \\
\hline & $\operatorname{SEm}( \pm)$ & 15.55 & 16.14 & 15.84 \\
\hline & D (at 5\%) & 46.20 & 47.94 & 47.07 \\
\hline & $\mathbf{I}_{1} \times \mathbf{M}_{0}$ & 1149 & 1150 & 1149.58 \\
\hline & $\mathbf{I}_{1} \times \mathbf{M}_{1}$ & 1797 & 1793 & 1794.78 \\
\hline & $\mathbf{I}_{1} \times \mathbf{M}_{2}$ & 1415 & 1406 & 1410.30 \\
\hline & $\mathbf{I}_{1} \times \mathbf{M}_{3}$ & 1670 & 1666 & 1667.88 \\
\hline & $\mathbf{I}_{2} \times \mathbf{M}_{0}$ & 1038 & 1053 & 1045.93 \\
\hline & $\mathbf{I}_{2} \times \mathbf{M}_{1}$ & 1794 & 1787 & 1790.67 \\
\hline & $\mathbf{I}_{2} \times \mathbf{M}_{2}$ & 1356 & 1352 & 1353.93 \\
\hline & $\mathbf{I}_{2} \times \mathbf{M}_{3}$ & 1626 & 1632 & 1628.89 \\
\hline & $\mathbf{I}_{3} \times \mathbf{M}_{0}$ & 1006 & 1032 & 1018.87 \\
\hline & $\mathbf{I}_{3} \times \mathbf{M}_{1}$ & 1607 & 1603 & 1605.03 \\
\hline & $\mathbf{I}_{3} \times \mathbf{M}_{2}$ & 1272 & 1271 & 1271.72 \\
\hline & $\mathbf{I}_{3} \times \mathbf{M}_{3}$ & 1353 & 1352 & 1352.23 \\
\hline $\mathbf{I} \times \mathbf{M}$ & $\operatorname{SEm}( \pm)$ & 26.93 & 27.95 & 27.44 \\
\hline & CD (at 5\%) & 80.02 & 83.04 & 81.53 \\
\hline
\end{tabular}


Table.5 Pearson's correlation coefficient (r) of yield contributing factors and corn yield with microclimatic parameters of baby corn affected by irrigation and mulching (pooled of two years)

\begin{tabular}{|c|c|c|c|c|c|c|c|c|c|c|c|c|c|c|c|}
\hline Characters & 1 & 2 & 3 & 4 & 5 & 6 & 7 & 8 & 9 & 10 & 11 & 12 & 13 & 14 & 15 \\
\hline 1. APAR 20 DAS & 1.000 & & & & & & & & & & & & & & \\
\hline 2. APAR 30 DAS & $0.340 * *$ & 1.000 & & & & & & & & & & & & & \\
\hline 3. APAR 40 DAS & 0.011 & -0.159 & 1.000 & & & & & & & & & & & & \\
\hline 4. APAR 50 DAS & 0.237 & -0.010 & 0.228 & 1.000 & & & & & & & & & & & \\
\hline 5. APAR 60 DAS & 0.261 & -0.252 & -0.185 & -0.076 & 1.000 & & & & & & & & & & \\
\hline 6. RUE 20 DAS & $-0.735 * * *$ & $-0.502 * * *$ & -0.034 & -0.168 & -0.059 & 1.000 & & & & & & & & & \\
\hline 7. RUE 30 DAS & -0.202 & $-0.710 * * *$ & 0.252 & $0.298^{*}$ & 0.163 & $0.564 * * *$ & 1.000 & & & & & & & & \\
\hline 8. RUE 40 DAS & $0.356 * *$ & 0.217 & $-0.516 * * *$ & 0.066 & 0.259 & -0.029 & -0.174 & 1.000 & & & & & & & \\
\hline 9. RUE 50 DAS & -0.182 & -0.195 & -0.014 & $-0.386^{* *}$ & 0.080 & $0.527 * * *$ & 0.077 & 0.134 & 1.000 & & & & & & \\
\hline 10. RUE 60 DAS & -0.182 & 0.125 & -0.269 & -0.134 & $-0.624 * * *$ & 0.261 & -0.073 & 0.128 & 0.075 & 1.000 & & & & & \\
\hline 11. Cob weight with husk & 0.155 & $-0.343 * *$ & -0.059 & 0.053 & $0.400 * *$ & $0.396 * *$ & $0.432 * * *$ & $0.477 * * *($ & $0.567 * * *$ & -0.087 & 1.000 & & & & \\
\hline 12. Cob length with husk & 0.088 & -0.001 & $0.521 * * *$ & $0.514 * * *$ & -0.209 & 0.160 & 0.199 & 0.226 & 0.196 & -0.012 & $0.411 * *$ & 1.000 & & & \\
\hline $\begin{array}{l}\text { 13. Cob weight without } \\
\text { husk }\end{array}$ & $0.310 *$ & -0.194 & -0.097 & 0.203 & $0.318^{*}$ & $0.309^{*}$ & $0.442 * * *$ & $0.547 * * *$ & $0.295^{*}$ & 0.0700 & $0.861 * * *$ & $0.430 * * *$ & 1.000 & & \\
\hline $\begin{array}{l}\text { 14. Cob length without } \\
\text { husk }\end{array}$ & 0.113 & -0.105 & $-0.512 * * *$ & $-0.325 *$ & $0.498 * * *$ & 0.159 & 0.204 & 0.237 & 0.185 & -0.010 & $0.407 * *$ & $-0.515^{* * *}$ & $0.377 * *$ & 1.000 & \\
\hline 15. Baby corn yield & 0.031 & 0.012 & -0.263 & -0.132 & 0.105 & $0.482 * * *$ & 0.076 & $0.533 * * *$ & $0.683 * * *$ & 0.2360 & $0.753 * * *$ & $0.358 * *$ & $0.741 * * *$ & $0.335 * *$ & 1.000 \\
\hline
\end{tabular}

Sample size $(\mathrm{N})=36$, Significant at $0.01 \%$ level (***), significant at $1 \%$ level (**), significant at $5 \%$ level (*) 
Fig.1 Effect of irrigation on total biomass accumulation $\left(\mathrm{g}\right.$ plant $\left.{ }^{-1}\right)$

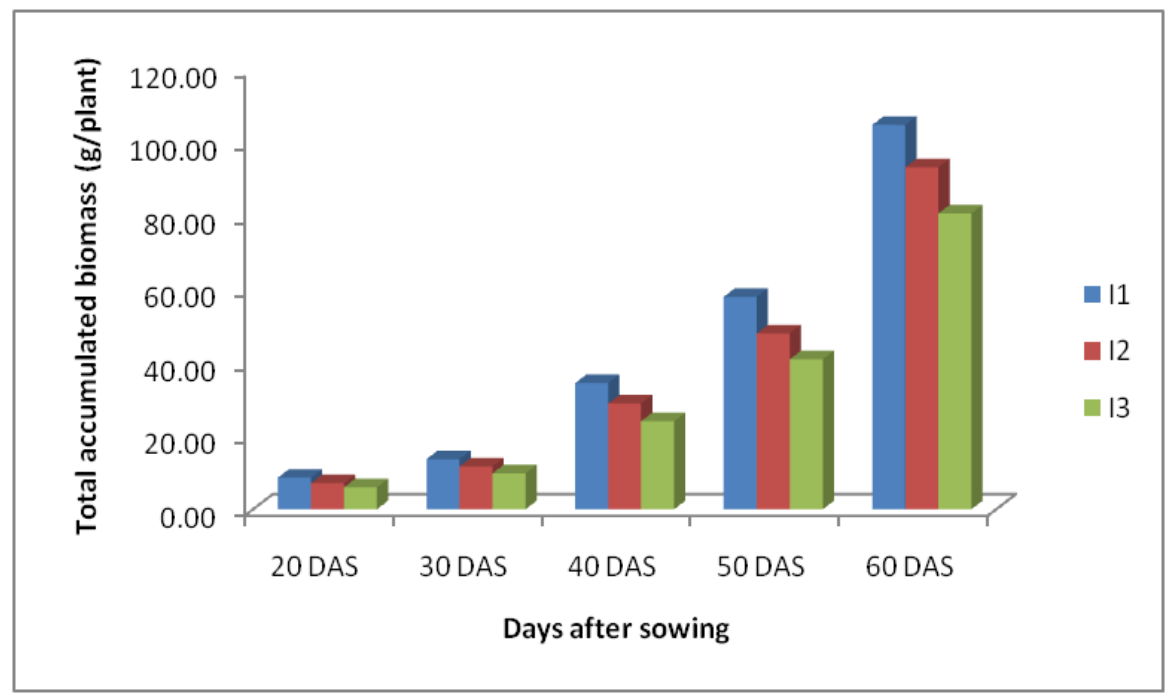

Fig.2 Effect of mulches on total biomass accumulation $\left(\mathrm{g} \mathrm{plant}^{-1}\right)$

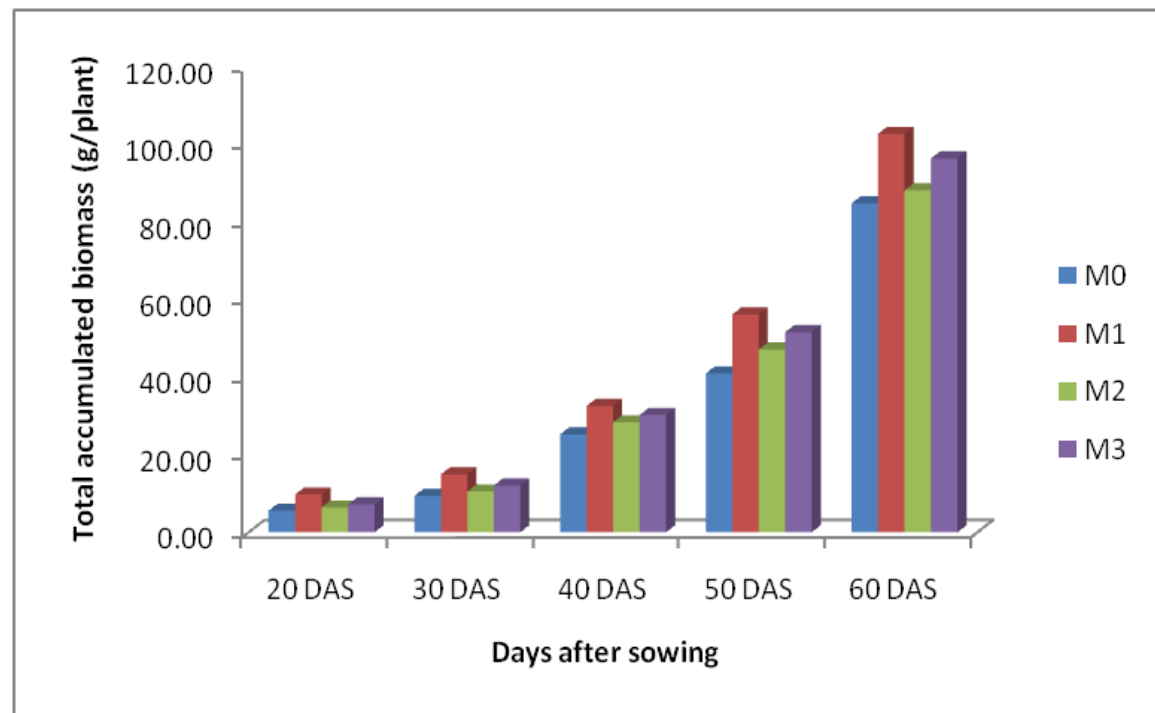

Yield attributing factors affected by irrigation and mulching of baby corn

Yield of baby corn very much depended on different yield attributing factors viz. Cob length $(\mathrm{cm})$, corn length $(\mathrm{cm})$, cob weight $(\mathrm{g})$ and corn weight $(\mathrm{g})$ and how irrigation and mulch have influenced all these parameters are discussed in Table 3. From the table it is clear that treatments with their interaction effect have significant effect on these factors.
Maximum cob length $(19.08 \mathrm{~cm})$, cob weight $(44.33 \mathrm{~g})$, corn length $(9.22 \mathrm{~cm})$ and corn weight $(10.75 \mathrm{~g})$ were achieved with sufficient application of water i.e. IW: CPE $1.0\left(\mathrm{I}_{1}\right)$ whereas minimum amount of all these factors were recorded with higher deficiency of water; IW: CPE $0.6\left(\mathrm{I}_{3}\right)$. More vigorous and luxuriant vegetative growth under sufficient water later increases the accumulation of higher biomass resulting higher yield production factor (Ertek and Kara, 2013). Yield attributes recorded 
maximum value (cob length, cob weight, and corn length and weight of $20.28 \mathrm{~cm}, 43.80 \mathrm{~g}$, $9.54 \mathrm{~cm}$ and $10.38 \mathrm{~g}$ respectively) by applying polythene mulch $\left(\mathrm{M}_{1}\right)$ and minimum in control plots $\left(\mathrm{M}_{0}\right)$. Mulch modified the crop microclimate, soil temperature thus higher accumulation of photosynthates resulted maximization of yield producing factors. These findings are in accordance with the results found by Yaseen et al., (2014). Individual treatments and their interaction effect $(\mathrm{I} \times \mathrm{M})$ resulted significant influence both the years.

Yield of dehusked baby corn affected by irrigation and mulching during 2016 and 2017

The data of dehusked cob yield achieved through the treatments are presented in Table 4. Baby corn yield was maximum $(1504.61 \mathrm{~kg}$ ha $^{-1}$ ) with IW: CPE $1.0\left(\mathrm{I}_{1}\right)$ and minimum (1311.96 kg ha-1) for IW: CPE $0.6\left(\mathrm{I}_{3}\right)$. In IW: CPE $1.0\left(\mathrm{I}_{1}\right)$, soil moisture always remain in field capacity, which resulted more efficient nutrient uptake, higher biomass accumulation with maximization of yield contributing factors. That significantly increased the cob and corn yield of baby corn. Similar results also reported by Shivakumar et al., (2011). The treatment with polythene mulch $\left(\mathrm{M}_{1}\right)$ recorded maximum corn yield (pooled) i.e. $1730.16 \mathrm{~kg} \mathrm{ha}^{-1}$ which were significantly superior over no mulch $\left(\mathrm{M}_{0}\right)$. All the growth and yield contributing factors are greater under mulched plots which contributed towards higher yield (Bhatt et al., 2004). The interaction effect of the treatments $\left(\mathrm{I}_{1} \times \mathrm{M}_{1}\right)$ recorded maximum corn yield of $1794.78 \mathrm{~kg}$ $\mathrm{ha}^{-1}$.

Correlation study of corn yield, yield forming characters with associated microclimate affected by irrigation and mulching during 2016 and 2017

The relevant data (pooled) of how the microclimate modified by irrigation and mulching have affected the yield attributes and yield of baby corn are presented in Table 5 and 6 respectively.

Cob yield with husk resulted negative correlation with APAR at 30 and 60 DAS. With LUE the association was positive, high positive correlation at 20 DAS but from 30 to 50 DAS, relationship was very high. Cob length with husk had very high positive correlation with APAR at 40 and 50 DAS. With RUE no significant relation was found. Cob weight without husk showed positive correlation with APAR at 20 and 60 DAS. RUE had high significant positive correlation with dehusked cob weight at 20 DAS and 50 DAS; in between at 30 and 40 DAS, the association was very highly positive. Cob length without husk had very high negative correlation at 40 DAS and 50 DAS with APAR; then at 60 DAS, the relationship was found to be highly positive. Baby corn yield was found to be correlated positively with RUE at 20, 40 and 50 DAS.

From the above research findings it can be concluded that applied treatments successfully modifies the crop microclimate to enhance better crop establishment, crop growth and yield. Judicious application of irrigation water with different mulches have significant role in increase of total crop biomass, better absorption of PAR, LUE which ultimately resulted higher yield forming factors of summer baby corn and finally increased cob and corn yield were recorded. The extent of modified microclimatic factors towards better yield forming factors and yield are well studied and described by the correlation among factors. So to save one of the major natural inputs in agriculture i.e. water by over using it as irrigation, it will be better to adopt climatological scheduling of irrigation (IW: CPE approach) with applying various covers or mulch to increase the moisture retention capacity of soil for longer duration. 


\section{Acknowledgement}

We, the authors express our gratefulness to Department of Agricultural Meteorology and Physics, B.C.K.V, Mohanpur, Nadia, W.B. for providing us necessary weather data from observatory and other instruments during the course of study.

\section{References}

Bhatt, Rajan.,Khera, K.L. and Arora, Sanjay. 2004. Effect of tillage and mulching on the yield of corn in the sub- mountainous rainfed region of Punjab. Int. J. Agric. and Bio. 6 (1):126-128.

Boonpradeep, S. and Chatasirt, M. 1999. Study on black gram cultivation in paddy fields under residue soil. In: Proceedings of National Moong bean Research Conference VII, Phitsanulok (Thailand), 1999, pp. 126-133.

Ertek, A and Kara, B. 2013. Yield and quality of sweet corn under deficit irrigation. Agricultural Water Management. 129: 138-144.

Gomez, K.A., and Gomez, A. 1984. Statistical Procedure for Agricultural Research, 2nd edn. John Willy and Sons, New York, USA, $680 \mathrm{p}$.

Han, H., Li, Z., Ning, T., Zhang, X., Shan Y., Bai M. 2008. Radiation use efficiency and yield of winter wheat under deficit irrigation in North China. Plant Soil Environment. 54: 313-319.

Jones, J.N., Moody, E. and Lillard, J. H. 1969.Effect of tillage, no tillage and mulch on soil water and plant growth. Agron. J. 61: 710-721.

Li, Q.Q., Chen, Y.H., Liu, M.Y., Zhou, X.B., Yu, S.L., Dong, B. D. 2008a: Effects of irrigation and planting patterns on radiation use efficiency and yield of winter wheat in North China. Agric. Water Manage. 95: 469-476.

Moitra, R., Ghosh, D.C., Sarkar, S. 1996. Water use pattern and productivity of rainfed yellow sarson (Brassica rapa L. var Glauca) in relation to tillage and mulching. Soil Tillage Res. 38: 153-160.

Müller, A.G., Bergamaschi, H., Bergonci, J.I., Radin, B., França, S., Silva, M.I.G. da. 2005.Estimating the leaf area index of maize crops through the sum of degreedays. Revista Brasileira de Agrometeorologia. 13: 65-71.

Narang, R.S., Nathusingh,Brar, R.S. and Muhal, S.S. 1989.Water management in winter maize grown on sandy loam soil.Indian Journal of Agronomy. 34(1):4-7.

Sahoo, S. C. and Mahapatra, P. K. 2007. Yield and economics of sweet corn (Zea mays L.) as affected by plant population and fertility levels. Ind. J. Agron. 52: 239-42.

Shivakumar, H.K., Ramchanrappa B.K., Nanjappa H.V. and Mudalagiriyappa. 2011. Effect of phenophase based irrigation schedules on growth, yield and quality of baby corn (Zea mays L.), Agric. Sci. 2 (3): 267-272.

Yaseen, R., Shafi, J., Ahmad, W., Shoaib, M., Salim, M. and Qaisrani, S.A. 2014. Effect of deficit irrigation and mulch on soil physical properties, growth and yield of maize. Environment and Ecology Research. 2(3):122-137.

\section{How to cite this article:}

Trisha Manna, G. Saha1, A. Saha, D. Dutta and Nanda, M.K. 2018. Contribution of Microclimate towards Yield Attributing Factors and Yield of Summer Baby Corn (Zea mays L.) under Different Irrigations and Mulches. Int.J.Curr.Microbiol.App.Sci. 7(07): 1542-1552. doi: https://doi.org/10.20546/ijcmas.2018.707.182 